Revue internationale P.M.E.

Économie et gestion de la petite et moyenne entreprise

\title{
Le polyformisme des entreprises et du territoire : une convergence possible des disciplines
}

\section{Cesare Emanuel}

Volume 2, numéro 2-3, 1989

URI : https://id.erudit.org/iderudit/1007936ar

DOI : https://doi.org/10.7202/1007936ar

Aller au sommaire du numéro

Éditeur(s)

Presses de l'Université du Québec

ISSN

0776-5436 (imprimé)

1918-9699 (numérique)

Découvrir la revue

Citer cet article

Emanuel, C. (1989). Le polyformisme des entreprises et du territoire : une convergence possible des disciplines. Revue internationale P.M.E., 2(2-3),

211-228. https://doi.org/10.7202/1007936ar
Résumé de l'article

Cet article porte sur les différents processus de transformation de la structure de production qui ont eu lieu dans l'Italie des années 1970 et 1980 à travers le concept de réseau. Ce concept permet en effet d'expliquer non seulement les diverses formes de réorganisation de l'entreprise industrielle, mais de relier cette réorganisation à l'évolution urbaine et territoriale. 


\title{
Le polyformisme des entreprises et du territoire : une convergence possible des disciplines
}

Cesare EMANUEL

Université de Verone

\begin{abstract}
RÉSUMÉ
Cet article porte sur les différents processus de transformation de la structure de production qui ont eu lieu dans l'Italie des années 1970 et 1980 à travers le concept de réseau. Ce concept permet en effet d'expliquer non seulement les diverses formes de réorganisation de l'entreprise industrielle, mais de relier cette réorganisation à l'évolution urbaine et territoriale.
\end{abstract}

\begin{abstract}
This paper examines the different processes used in Italy during the 1970 s and 1980 s to transform the structure of production through the concept of network. This concept may be used not only to explain the various forms of reorganizing industrial enterprises, but also to link this reorganization to urban and territorial development.
\end{abstract}

\section{RESUMEN}

Este articulo trata sobre los diferentes procesos de transformacion de la estructura de produccion que ha tenido lugar en Italia desde los anos 70 y 80 atravez del concepto de red. Este concepto permite en efecto explicar no solamente las diversas formas de reorganizacion de la empresa industrial, si no tambien de asociar esta organizacion a la evolucion urbana y territorial.

- Cesare Emanuel est chercheur à la Faculté d'économie de l'Université de Verone. Ses recherches et ses publications portent en particulier sur la géographie urbaine, la géographie économique et la cartographie thématique. Adresse: Facolta' di Economia e Commercio - Istituto di Geografia, Via dell'Artigliere, 19-37100. 


\section{Introduction}

A partir de la première moitié des années 1970, le panorama économique, social et territorial de l'Italie s'est profondément transformé par rapport aux premières décennies de l'après-guerre. Il est d'ailleurs évident que ces transformations peuvent être définies comme structurelles, car elles ont marqué les aspects quantitatifs et, surtout, qualitatifs du développement (l'organisation, les styles de vie, les comportements, etc.).

L'analyse spatiale a défini progressivement ces transformations par des termes tels que désurbanisation, décentralisaiton, désindustrialisation, urbanisation diffuse, etc. Pourtant, ces termes n'ont que très rarement permis d'exprimer la complexité profonde des interactions de tous les phénomènes en cause. Ce n'est pas par hasard que le terme de désindustrialisation a été associé à la crise productive des zones métropolitaines (et donc à leur déclin démographique), plutôt qu'aux changements dans l'organisation des entreprises et dans la production.

Une telle lacune provenait en partie des difficultés de nombreuses disciplines qui traitent de cette problématique (l'économie, la géographie, l'urbanisme, la sociologie, etc.) à développer l'approche inter-disciplinaire nécessaire. Mais, dans les dernières années, de nombreux points de contacts se sont noués entre ces disciplines et la notion du réseau a pu ainsi s'affirmer; même si cette notion possède des significations différentes, elle apparaît maintenant dans toutes les disciplines citées. Dans les recherches en géographie régionale, cette notion est employée de plus en plus souvent pour définir la structure des espaces régionaux. Par contre, dans l'analyse économique et dans la sociologie de l'organisation, elle se rapporte aux relations entre les diverses unités de production et aux liens de ces dernières avec les autres aspects sociaux et institutionnels.

Dans l'interprétation de l'espace économique, ces deux approches semblent être d'ailleurs fort complémentaires; d'où la nécessité d'essayer d'illustrer les liens logiques entre la dimension économique et fonctionnelle et celle, géographique, du concept du réseau.

Pour cela, on doit passer systématiquement de l'analyse conceptuelle à l'analyse empirique, et réciproquement, pour une approche «éclectique» des données, à des problématiques et des théories pertinentes avec l'objectif. Tout d'abord, on s'arrêtera sur les transformations les plus importantes de la structure productive, sur les facteurs qui ont permis ces dernières et sur l'identification d'un éventuel modèle macroéconomique de référence. Ensuite, on analysera quelques modèles élémentaires de réseaux d'entreprises industrielles et leurs liens logiques pouvant servir de jonction avec les réseaux territoriaux. On présentera enfin les caractéristiques et les typologies des réseaux urbains contemporains et leurs relations possibles avec les réseaux d'entreprise. 


\section{Le contexte}

Dans l'évolution de la structure manufacturière italienne, on a assisté, à partir de ces années 1970, à un phénomène absolument imprévu dans les décennies précédentes, soit l'apparition d'un nombre considérable d'entreprises aux dimensions souvent très réduites (100000 seulement dans la décennie 1971-81). Ces entreprises ont contribué en bonne partie à compenser la baisse des emplois dans les grandes entreprises et à générer de nombreux nouveaux postes de travail (600 000 environ). Cette évolution n'était pourtant pas accidentelle; cette multiplication des petites entreprises s'intégrait harmonieusement, soit à la structure sectorielle, soit à la structure territoriale de tout l'appareil productif (Tassinari, 1984; Cori, 1985).

En effet, d'une part les PME se sont développées dans des secteurs désormais consolidés de la structure de production sans y apporter des changements décisifs. D'autre part, cette multiplication des PME s'est faite un peu partout, soit au nord-ouest du pays (où la grande entreprise s'est le plus développée), soit dans les zones traditionnelles de croissance (c'est-à-dire la troisième Italie), soit enfin dans les régions moins industrialisées du sud.

Ce taux élevé de création d'entreprises s'est maintenu même dans les périodes récentes, et simultanément avec le retour de la croissance de la productivité dans les grandes entreprises (Garofoli, 1891). Cette tendance, mesurée non seulement entre les divers recensements mais aussi à l'aide d'analyses intersectorielles, présente une forte diversification et surtout une complexité et une interdépendance croissantes entre les divers secteurs de l'économie (Foloni, 1984). Le résultat en est une faible polarisation dans les secteurs les plus traditionnels de l'économie.

Dans les limites de l'analyse «input-output», on peut trouver d'autres relations plus riches, avec divers niveaux de relations et qui montrent que les secteurs les plus stratégiques ne sont plus uniquement ceux «de pointe», mais aussi des activités tertiaires intervenant dans les phases intermédiaires ou finales du cycle de production.

Tous ces aspects semblent confirmer que cette «troisième Italie» se développe aussi dans les autres parties de l'Italie, c'est-à-dire tant dans les zones des grandes entreprises que dans celles peu développées où s'affirment actuellement de nouveaux modes de production et d'organisation ainsi que de nouvelles formes de gestion des ressources employées (Regini et Sabel, 1989).

Pour bien comprendre cela, il faut évidemment changer l'unité d'observation et utiliser des points de référence qui prennent pour base analytique et conceptuelle une unité qui n'est plus l'entreprise unique (Lorenzoni, 1985). Ce passage «de l'entreprise aux entreprises» peut être vu, en termes linguistiques, comme le fait de passer de «l'un au multiple» et de ramener aux traits distinctifs deux modèles organisatifs différents (Costa, 1986).

«L'un», du moins en Italie, indique la grande entreprise et ses mécanismes de développement et de régulation. Il constitue une tentative, qui fut d'ailleurs réussie en particulier dans les années 50 et 60 , d'obtenir des productions importantes grâce 
à une intégration verticale et des économies d'échelles dans de grands établissements. Il est caractérisé par une stabilité de la production qui domine le marché et par de grands volumes à faibles coûts généraux et unitaires et, par conséquent, à faibles prix. Dans ses éléments essentiels, le modèle suppose donc un contrôle centralisé et hiérarchique de l'entreprise, avec une gestion tendant à éliminer l'incertitude et la précarité de la production et de la demande intérieure et extérieure. Le contrôle de l'innovation, de la technologie, des ressources et du capital dans des productions standardisées explique l'efficacité de la grande dimension et relègue les petites entreprises aux fonctions résiduelles et peu favorables. Cette économie bipolaire associe les grandes firmes aux secteurs modernes et laisse aux petites entreprises les productions traditionnelles (Vacca, 1985).

«L'un», on le sait bien, n'intéresse pas seulement l'économie, mais aussi le territoire. Le modèle de la «factory town», où la forte concentration des entreprises, des ressources productives, de la main d'oeuvre et du contrôle permanent de l'espace urbain crée, avec ses forces centripètes, les grands déséquilibres territoriaux tant dans les régions qu'au niveau national, le représente bien (Borlenghi et Dematteis, 1982).

«Le multiple», par contre, constitue dans un certain sens son antithèse.

À mesure que les économies de «l'un» et des grandes agglomérations deviennent moins «économiques» pour de larges secteurs d'activités, la restructuration ou la correction deviennent nécessaires. On arrive ainsi à une «polyformie» des secteurs et des espaces qui se reflète dans le développement des économies d'échelle au niveau de l'entreprise ou du système d'entreprises.

La diversification croissante ne signifie pas seulement la crise du modèle centralisé, mais elle se révèle importante pour créer des services capables de développer la technologie et l'information, en plus de favoriser la coordination permettant des économies importantes de champ et de variété (Senn, 1988).

«Le multiple» favorise l'interdépendance croissante entre le secteur manufacturier et le secteur tertiaire, sous forme de machines, de technologies, d'opérateurs de contrôle et de gestion. Ainsi, il freine la tendance à la désindustrialisation. L'informatisation renforce cette interdépendance; et le savoir-faire technique s'oriente non seulement vers l'innovation de produit, mais aussi vers l'amélioration systématique des processus et des organisations avec, comme conséquence, l'abaissement des coûts de production et le déplacement du caractère central de l'entreprise de production vers des institutions et des «réseaux» (Cappelin, 1989).

Dans une certaine mesure, tout cela correspond à une spécialisation croissante des opérations qui affecte non seulement les phases ou les secteurs de l'entreprise mais aussi le territoire, et qui permet d'améliorer rapidement l'adéquation, sous forme de différentiation et d'une nouvelle programmation, en temps réel, des biens avec la demande.

Dans le "panier de consommation», on trouve des produits beaucoup plus spécialisés qui répondent aux différentes demandes des consommateurs, quels que 
soient leurs revenus ou leur niveau d'information. «Le multiple» s'associe donc aux nouveaux langages et à la nouvelle éthique que la recherche sociologique recherche dans le cheminement qui mène du modèle social de type mécanique à celui de type organique (Butera, 1986).

\section{$3 \quad$ Les modèles élémentaires}

La création de nouvelles entreprises et le déplacement d'une bonne partie d'entre elles dans les zones traditionnellement périphériques (par rapport aux «premiers foyers» d'industrialisation) ont été souvent analysés (et trop simplifiés) en termes de décentralisation productive et territoriale. Ces termes englobaient à leur origine des phénomènes larges et différents de «recentralisation» rarement précisés, tels que des stratégies de diversification, de spécialisation ou d'intégration qui cherchaient à redéfinir l'ensemble des relations existant dans l'espace, dans les secteurs mêmes et entre ces derniers (Camagni, 1989).

Éclaircir ces liens signifie appréhender encore plus les réseaux et le type de relations qu'ils évoquent ou produisent. Un tel cheminement est complexe. A l'aide de la méthodologie de R. Brunet (1980), déjà expérimentée pour l'analyse et la représentation des structures de l'espace et des relations, les manifestations plus importantes de tels processus devront être décomposées en modèles élémentaires, valables pour les cas spécifiques, et recomposées ensuite en modèles plus génériques pouvant être appliqués au niveau régional ou national.

En acceptant de simplifier la réalité et en utilisant l'idée de Boldisser (1984), on peut regrouper les différentes formes de décentralisation et de réorganisation en quatre types : la décentralisation «de capacité», la désintégration verticale, la division du travail entre les entreprises et la délocalisation des entreprises. Dans cette délimitation, ces quatre types peuvent être considérés comme des modèles de l'espace élémentaires, ou encore comme les stratégies particulières d'évolution des entreprises. Ils impliquent la mobilité totale ou partielle et/ou la réorganisation des relations internes ou externes dans et entre les entreprises.

\subsection{La décentralisation "de capacité»}

C'est la forme de décentralisation la plus traditionnelle, effectuée dans le but de rechercher la flexibilité des facteurs de production (en particulier du travail, selon les différences de salaires ou de rémunération professionnelle).

Le noyau central qui dirige et organise les sous-systèmes périphériques détermine le caractère hiérarchique de cette forme de décentralisation; celle-ci est gérée au moyen de codes, de règles et même de procédures télématiques d'avant- 
garde (contrats de franchises, contrôles de qualité, etc.) ou peu formalisées (ordres univoques et standardisés, stables au cours du temps).

Comme il s'agit d'une rationalisation pour la plupart du temps interne à l'entreprise, elle n'est pas particulièrement importante ni significative dans les processus de restructuration industrielle, même s'il ne manque pas d'exemplesimportants, tels les «hollow corporations» qui se développent actuellement. La représentation de ce modèle prévoit l'existence d'un noyau central (siège-social) et d'un faisceau des vecteurs radiaux totalement convergeants et dépendants du centre, aux extrémités périphériques où se trouvent les unités d'opération. En Italie, au début des années 1970, cette forme de réorganisation a créé une «méridionalisation» du capital industriel.

\subsection{La désintégration verticale}

Les modalités de cette réorganisation ressemblent aux précédentes. Mais, dans ce cas, les divers noyaux techniques et productifs deviennent autonomes et décentralisés. Ils permettent ainsi la création de nouvelles entreprises qui, tout en étant juridiquement séparées, restent quand-même contrôlées par l'entreprise-mère, devenue le chef de groupe ou le holding financier du système ou du groupe industriel.

Les entreprises décentralisées jouissent d'une autonomie naturelle. Elles peuvent maintenir leurs relations avec l'extérieur, développer des collaborations ou des sous-traitances, et même des conventions entre elles et les unités locales dans les limites de territoires décentralisés.

Par conséquent, plus la taille et la complexité des sous-systèmes s'agrandit, plus grande est la probabilité que les systèmes subordonnés deviennent autonomes dans leur effort de spécialisation et d'autogestion.

L'entreprise orientée vers la désintégration verticale peut être représentée par diverses formes multipolaires. Le modèle peut être ramené à l'entreprise-réseau (figure 1,p. 218); l'organisation des fonctions et de l'espace peut être représentée à l'aide d'un système solaire (le «solar system entreprise» de Piore et Sabel) dominé par des états-majors capables de gouverner les facteurs critiques et stratégiques du développement de l'entreprise.

Autour du centre se trouvent les pôles d'opération constitués par des entreprises ou par des fonctions qui se spécialisent et auxquelles se rattache l'univers de la micro-entreprise répartie sur le territoire. C'est la réorganisation qui se produit actuellement dans les grandes firmes italiennes des secteurs de l'automobile et du textile et qui intéresse surtout les zones «centrales» du système (le Nord Ouest).

\subsection{La division du travail entre les entreprises}

Al'aide de ce type, on entend les relations de collaborationà moyen età long termes entre les groupes d'entreprises autonomes qui réalisent des productions conjointes, selon des procédures spécifiques de concertation. Ce modèle, dans la 
«troisième Italie», concerne les secteurs traditionnels, alors que dans les grandes zones métropolitaines, il touche les secteurs avancées.

Entre les unités, un réseau dense d'interdépendances s'établit. Il est généré par les entreprises, même de petites tailles. Les motifs pour lesquels celles-ci sont encouragées à se partager les différentes activités peuvent être très variés. Ils vont de la recherche d'une production spécialisée qu'une seule entreprise n'est pas capable de réaliser au développement d'économies d'échelle importantes. Dans ce dernier cas, chaque unité se spécialisera dans une production plus appropriée à sa structure organisationnelle et technico-productive. Alors que dans le premier cas, il s'agit d'une réorganisation fondée sur les rapports de marché, dans le second cas, les rapports entre les entreprises s'effectuent au moyen d'accords précis de coopération qui s'étendent du consortium commercial d'offre aux associations de type partenariat («joint-venture») (Camagni et Gambarotto, 1985).

Ce n'est que dans les dernières années que cette forme d'organisation s'est répandue, créant des effets de synergie pour tout le système industriel. L'autonomie relative des entreprises, la capacité complémentaire développée et l'égalité hiérarchique entre les unités individuelles de cette forme d'organisation ont permis l'apparition de plusieurs néologismes tels que «réseaux d'entreprises, entreprises sans centre, constellations d'entreprises, etc.» Dans cette organisation, on trouve toujours une entreprise-guide; mais, en général, on suppose que la stratégie globale dérive du réseau dense de relations plus ou moins égalitaires entre les diverses unités.

Comme ce système s'organise directement entre les entreprises, sa base est explicitement territoriale. L'extension géographique de ce réseau pourra se manifester soit vers les centres, soit vers d'autres périphéries, ou vers les deux à la fois. Cependant, l'accessibilité aux sources et types d'information aux types d'activités impliquées ou à la quantité et à la qualité des innovations réalisées et/ou produites par les systèmes individuels du réseau pour la base territoriale peut être, selon le cas, fort différente.

Ce modèle peut se décomposer selon ses fonctions; soit : a) un modèle à interaction tertiaire dominante, où les noeuds du réseau sont constitués par la direction des entreprises-guides, par des services de $\mathrm{R} \& \mathrm{D}$, de marketing, de financement ou des entreprises de haute technologie, etc. b) un modèle où les entreprises productives sont traditionnelles et parallèles aux services de base complémentaires. Le premier cas pourrait correspondre à la typologie du parc-scientifique (Giacometti, 1986), le second aux zones-système, comme on peut le voir à la figure 2 (p. 219).

\subsection{La délocalisation}

La délocalisation de tout le complexe d'entreprises, du centre vers la périphérie, représente enfin une réorganisation du type économico-social qui se distingue de la réorganisation technique discutée en 3.1 et 3.2. 
Fig. 1 Entreprises-réseaux

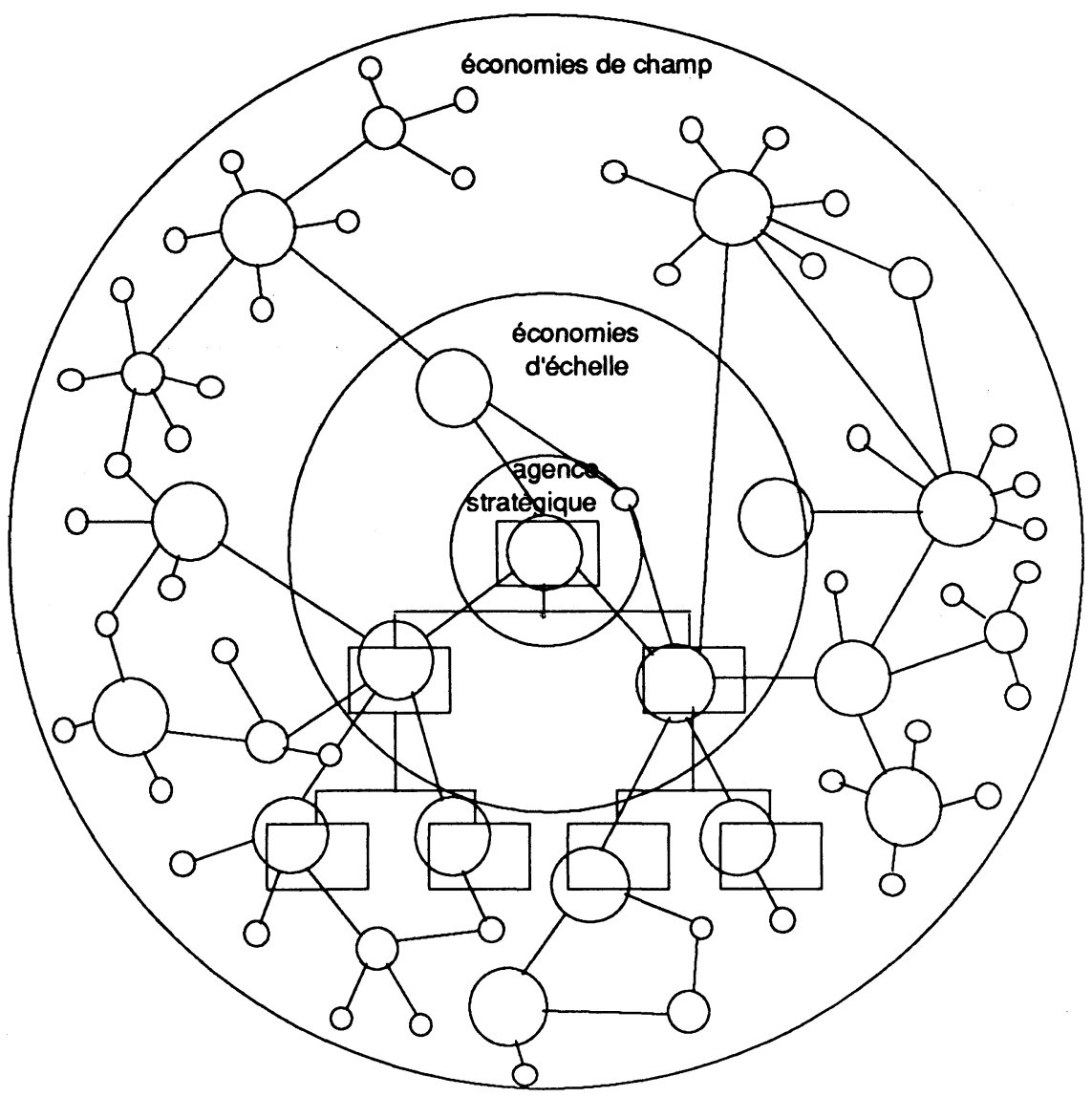

Source : Lito, (1989), (déjà reproduit par Butera).

Ce modèle est très simple. Il suppose le déplacement tant des fonctions productives que de celles des directions d'entreprises. Il s'applique rarement dans le cas des grandes entreprises, mais il est assez courant dans le cas des PME. Dans les dernières cinquante années, en Italie, on a assisté toutefois à des relocalisations de tout un noyau de grandes entreprises vers de nouvelles zones urbaines, ou, plus récemment vers des zones sub-urbaines métropolitaines, (Aiken, Martinottie tStrickland, 1987). 
Les modèles que nous venons de discuter sont évidemment simplifiés. La réalité présente des comportements plus disparates, comme par exemple des situations où la décentralisation et la relocalisation en plusieurs lieux des fonctions peuvent être assumées par des bureaux et des magasins. De plus, ces modèles ne touchent qu'aux formes inverses de réorganisation de l'espace telles la centralisation ou la décentralisation et la recentralisation «implicite» (par exemple la récente acquisition des actions de la Montedison par Gardini a obligé cette firme à se relocaliser dans la ville de son acquérant). Ces cas de centralisation ou de recentralisation sont assez fréquents si l'on observe le cycle complet de l'activité productive, en incluant les phases de commercialisation. Cette fonction commerciale peut même imposer le regroupement de tout un complexe productif. Récemment, le nouveau besoin des productions «à demande» («just in time») est en train de révolutionner toute la distribution en favorisant le retour au «centre» de phases entières de productions délocalisées depuis peu de temps (Senn, 1989).

On voit aussi des cas où l'entreprise doit se recentraliser ou se décentraliser «implicitement» sans changer de place, à cause de l'expansion ou du déclin fonctionnel du centre urbain dans lequel elle se trouve. Ce modèle de réorganisation représente une forme de flexibilité indirecte, semblable à celle qui se produit dans le cas de systèmes rigides de production (constitution de stocks ou production des biens pour une demande très cyclique ou variable, extension temporaire des marchés, etc.) (Arrighetti, 1988).

Fig. 2 Le modèle d'une aire-système

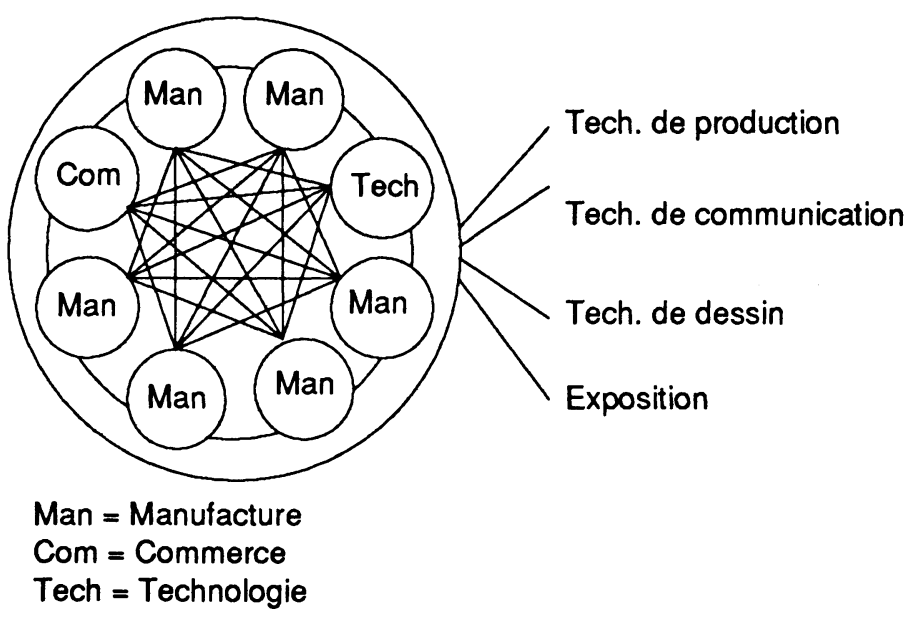

Source : Camagni et Rabelloti, (1988). 


\section{$4 \quad$ Les noeuds du réseau : les systèmes territoriaux à développement endogène}

L'analyse conjointe de ces formes élémentaires de réorganisation permet de concevoir un modèle général fortement fragmenté et qui évoque l'idée d'une «immense usine diffuse» constituée d'un réseau dense et complexe d'interdépendances entre de petits noeuds locaux ouverts vers l'extérieur et, comme on l'a déjà vu, très instables. Il est donc facile de voir comment le développement et l'évolution économique, technologique et de marché fonctionnent en tant qu'agents de déstructuration de l'ancien équilibre, en constituant un espace non plus homogène, mais réticulaire.

Il va de soi, cependant, qu'une simple transposition mécanique des réseaux d'entreprises sur le territoire, sans concept intermédiaire plus large, poserait des problèmes de lecture; c'est-à-dire que les phénomènes en questions manqueraient d'une nécessaire transparence et seraient, en plus, difficilement mesurables.

L'espace réticulaire générée par ces formes de réorganisation produit donc un espace virtuel dans lequel le territoire apparaît plutôt comme un récipient neutre et non un "producteur» actif dans le développement des relations et des synergies.

Les catégories conceptuelles les plus pertinentes pour le passage des réseaux d'entreprises aux réseaux territoriaux semblent être celles qui permettent de préciser les caractèresendogènes et exogènes de l'environnement local(Dematteis, 1989a etb).

Effectivement, on peut avoir à faire face à un développement endogène lorsque les entreprises, ou les établissements d'entreprises réparties sur le territoire sont capables d'établir, avec ce dernier et avec des effets de synergies traduisibles en caractéristiques réciproques, des spécialisations ou d'autres formes d'organisation évolutive. Ces formes de développement particulier, capables même de créer des différentiations géographiques, s'opposent à celles qui sont produites par le développement exogène, dans lequel les liens entre l'environnement externe et les entreprises sont faibles, même si la dépendance extérieure est forte. Dans ce dernier cas, le potentiel d'innovation, avec ses implications en termes de gestion interne et d'évolution historique culturelle, ainsi que le potentiel génétique pour la reproduction seront limités.

Il en résulte que pendant que les centres à développement endogène prédominant parviennent à constituer des noeuds forts interreliés fonctionnellement aux réseaux territoriaux (dans ce cas, les grands espaces métropolitains ou les districts industriels périphériques consolidés), les centres qui génèrent une croissance du type exogène pour la plus grande partie tendent à demeurer plus flous et moins développé dans le substrat réticulaire relativement indéfini par l'industrialisation «diffuse». Alors que dans le premier cas, il s'établit un rapport biunivoque fort entre le territoire et l'entreprise, dans le second, le territoire devient en bonne partie un support neutre, et ses spécificités tendent alors à rester liens plutôt que ressources. 
Selon l'échelle géographique d'observation adoptée, ces noeuds de caractère endogène peuvent devenir plus nombreux ou même disparaître, en donnant une forme aux structures et aux problématiques particulières. Différencier ces réseaux territoriaux devient donc une opération complexe qui ne peut être définis par des grandeurs physiques mesurable objectivement.

Dans beaucoup d'études, l'identification des divers types de spécialisation fonctionnelle et de solidarité territoriale a produit différentes typologies de réseaux territoriaux qui, dans le cas italien, peuvent être regroupées ainsi :

Les réseaux de districts technologiques (Antonelli, 1986). Actuellement, ce type semble constituer la forme la plus évoluée des réseaux territoriaux (les technopoles émergentes du Nord-Ouest). Dans ses noeuds, on observe une interaction prédominante entre les activités de direction, les activités de $\mathrm{R} \& \mathrm{D}$ des grandes entreprises, les activités de services spécialisés externes (finances, marketing, ingénierie, etc.) et les activités qui possèdent un contenu technologique plus élevé que dans les entreprises manufacturières.

Dans les noeuds de ce réseau, les processus importants de recentralisation sélective conduisent à des formes d'organisations agglomérées, à des parcs scientifiques, à des districts industrielset des infrastructures polarisantes du type métropolitain.

Les liens entre ces nouds se manifestent non seulement sur le plan technoscientifique, mais aussi sur le plan manufacturier et financier, à travers un réseau relationnel fortement interrelié, et enfin dans un marché du travail et un contexte morphologique de l'environnement très évolué.

Les effets synergiques se manifestent donc sur le plan de la conception et de la production d'innovations, mais aussi sur celui de l'incubation et de la regénération continue, à travers les fréquents échanges commerciaux avec d'autres systèmes extérieurs.

Les réseaux situés autour de grands établissements à caractère local (Maillat, 1989). Il s'agit d'une variante des districts technologiques où les forts noeuds sont constitués par les sièges sociaux de grandes entreprises qui maintiennent des liens importants avec le territoire d'où elles proviennent et des unités de production qui appartiennent non seulement au secteur manufacturier mais aussi aux services spécialisés et aux services de support à la production et à la commercialisation. À travers une spécialisation progressive, ces dernières firmes peuvent devenir en grande partie indépendantes de la grande entreprise, et ainsi, moins sujettes aux fluctuations locales et plus ouvertes aux interactions avec les systèmes extérieurs. Le modèle du réseau correspond donc à la somme de l'accroissement des réseaux d'entreprises et d'entreprises-réseau. L'autonomie relative acquise par certaines unités produit pourtant une autonomie parallèle et une spécialisation des noeuds territoriaux dans lesquels ils sont insérés.

Les réseaux des zones-systèmes et des districts industriels (Garofoli, 1986; Becattini, 1989). Cette typologie est, encore une fois, proche de celle décrite précédemment, alors que l'élément distinctif est avant tout représenté par une 
présence très riche de PME dans le même secteur et par l'utilisation des ressources presque uniquement locales pour la production. Ici, la division très accentuée du travail entre les entreprises entraîne une spécialisation croissante et des liens complexes qui vont jusqu'au développement d'entreprises produisant des équipements pour elles. Les relations entre les noeuds sont donc de type soit infrasectoriel, soit intrasectoriel.

Ce type de développement est vu comme autocentré; c'est-à-dire qu'il est basé sur des entreprises, des technologies, du capital et du travail et des technologies presque entièrement locaux, alors que très peu d'unités de production appartiennent à des entreprises externes. Cela signifie que le réseau local est en mesure d'orienter son propre processus de développement et de transformation;

Les réseaux de systèmes de production locaux (Garofoli, 1986). Ce réseau est aussi caractérisé par la présence dominante de petites entreprises spécialisées dans le même secteur; beaucoup d'entre elles produisent donc le même bien. Les relations entre les noeuds sont donc fortes même si, pratiquement, elles se limitent aux liaisons de type sectoriel. Le système, dans sa complexité est bien structuré et repose historiquement sur une culture technico-professionnelle particulière qui permet de contrôler les modifications dans les processus de production avec des ajouts techniques marginaux;

Les réseaux de zones de productions spécialisées (Garofoli, 1986). Ce type de réseau territorial se caractérise, lui aussi, par une prédominance de PME. Sa structure est de type horizontal, car toutes ces entreprises produisent le même type de marchandise ou effectuent la même phase de travail et sont donc concurrentes sur le même marché. En général, les réseaux de ce type sont de formation récente, souvent générés par la décentralisation d'un réseau territorial, avec prépondérance d'entrepreneurs extérieurs ou d'installations de productions dépendant d'entreprises à l'extérieur de la zone considérée (la structure, dans ce cas, tend à évoluer vers des formes exogènes de développement);

Les réseaux résultant d' une "verticalisation» des grandes entreprises (Maillat, 1989). Leur formation dérive de stratégies de réorganisation des grandes entreprises, comme cela a été étudié dans l'analyse des «modèles élémentaires». Dans quelques cas, d'ailleurs peu nombreux en Italie, la «réorganisation» simultanée des processus productifs de beaucoup d'entreprises peut mener à la formation d'un réseau de synergies locales, capables de générer un développement «autopropulsif» et accentuer l'indépendance de ces unités par rapport aux entreprises-mères.

Dans ce cas, il s'agit d'une forme originale du passage de stratégies de «filiation» d'entreprise aux réseaux d'entreprises, où les ressources territoriales (dans un réseau assez dense de centres accessibles et de fonctions urbaines adéquates) jouent un rôle important.

Les réseaux territoriaux analysés ci-dessus présentent des «solidarités» importantes à l'échelle régionale et locale. Récemment, des réseaux synergiques 
extra-régionaux stables se sont consolidés non seulement à cause du développement d'activités «de pointe» et de haute direction, mais aussi de productions moins avancées appliquées toutefois à des produits nouveaux ou de haute gamme. Dans ce cas, les synergies seraient créées par les liens entre les entreprises spécialisées dans la sous-traitance complètes et complexes et les entreprises-guide faisant partie d'oligopoles mondiaux (Esposito, Raffa et Zollo, 1989).

C'est donc une forme de «globalisation» relative des spécialisations locales, capable non seulement d'insérer les économies locales dans des noeuds complexes les plus avancées mais aussi de rendre la structure des relations locales de plus en plus stable.

\section{$5 \quad$ Les réseaux fonctionnels et physiques de la ville}

Les entreprises tirent donc leur dynamisme de leur appartenance à un réseau. Ces entreprises et le territoire ne sont pas deux sujets séparés, mais l'un est en partie le produit de l'autre. Comme nous avons pu le constater, si le territoire joue un rôle d'incubateur, surtout pour les entreprises les plus dynamiques, on peut se demander comment la diffusion et la réorganisation de la production influencent le dynamisme même de l'organisation territoriale. Sur cet aspect de l'analyse, nous n'avons pas d'explication claire.

L'analyse fonctionnelle du territoire a depuis longtemps mis en évidence comment le réseau hiérarchique était souvent un produit plus abstrait que social, découlant de l'application rigide du modèle des localités centrales (Da Pozzo, 1979). Après la guerre, les réseaux urbains se sont, en effet, polarisés à un haut degré; à l'intérieur de ceux-ci, les distinctions entre les différents types de centres ont perdu une grande partie de leur capacité explicative (Vallega, 1979).

Il demeure qu'actuellement, les phénomènes de réorganisation de la production rendent beaucoup plus difficile la délimitation de chaque système urbain. Les études les plus récentes (Dematteis, Gambino et Coppola, 1986; Landini, 1987; Celant, 1988; Bernardi, 1988) ont effectivement démontré que les changements discutés plus haut transformeraient les réseaux précédemment «arborescents» (figure 3-a, p. 225) ou qui correspondent à des relations à plusieurs niveaux — ou les réseaux à étoile (figure 3-b) où l'on trouve une forte polarisation régionale - en réseaux fortement interconnectés (figures 3-c) et qui correspondent à une situation de décentralisation exogène ou de centralisation endogène modifiant en partie la structure précédente arborescente (figure 3-d).

Autrement dit, les causes qui ont amené toutes les différences dans le processus d'industrialisation diffuse de l'organisation à réseau d'entreprises peuvent expliquer aussi ici une différence entre les systèmes urbains en tant que réseaux, à 
l'intérieur duquel les liens hiérarchiques entre chaque centre perdent de leur force et où l'espace présente une discontinuité beaucoup plus large. Dans ce sens, un centre particulier ne reconstitue le plus souvent qu'une partie d'une organisation plus complexe et plus large (Emanuel, 1989). Ces transformations expliquent pourquoi on en arrive à un polyformisme territorial de plus en plus fort, polyformisme accompagné d'innombrables formes d'urbanisation et de paysages urbains (Petsimeris, 1989).

Tout cela nous ramène encore une fois au «coeur» des processus de réorganisation fonctionnelle. Là où les districts technologiques et les directions centrales s'affirment et se développent, le rapport entre les ressources endogènes et exogènes évolue très rapidement; le réseau de relations tend non seulement à se renforcer localement, mais aussi à se relier à d'autres noeuds spécialisés et stratégiques, sur un échiquier qui dépasse les limites des régions et des nations. Ces noeuds urbains tendent donc à se différencier du reste du territoire et à assumer des positions dominantes. C'est avant tout dans ces milieux que la capacité compétitive des systèmes nationaux se mesure (Conti et Spriano, 1989).

Mais c'est aussi dans les noeuds régionaux restants que les capacités d'opération et d'expansion des relations entre les industries évoluent. C'est à ce second niveau du réseau du système urbain territorial que les réseaux des districts ou des systèmes locaux de production (et la désintégration verticale des grandes entreprises) entraînent constamment un repositionnement des centres.

Les zones les plus marginales restent exclues de ces bouleversements; leurs réseaux y sont soit indifférents, soit dirigés de l'extérieur. C'est dans ces zones, qui du point de vue de la géographie sont «interstitielles» aux grands systèmes urbains, qu'on peut voir des régressions de capacités de production et le déclin économique et social du territoire.

\section{Conclusion}

Cette analyse a voulu montrer la pertinence de la théorie des réseaux. Elle soulève toutefois beaucoup de questions, surtout de nature théorico-méthodologique. Cependant, au lieu de s'arrêter à ces zones d'ombre, il convient de résumer schématiquement les notions discutées.

$1^{\circ}$ Une première certitude : l'entreprise structure et déstructure le territoire.

Si cette constatation est implicite dans les études économiques, elle ne l'est presque jamais dans les disciplines touchant au territoire : pour elles, les principales transformations urbaines s'expliquent généralement par les services tertiaires, considérés comme les principaux responsables de la centralité urbaine et de ses trajectoires évolutives. L'entreprise manufacturière et ses fonctions 
Fig. 3

A

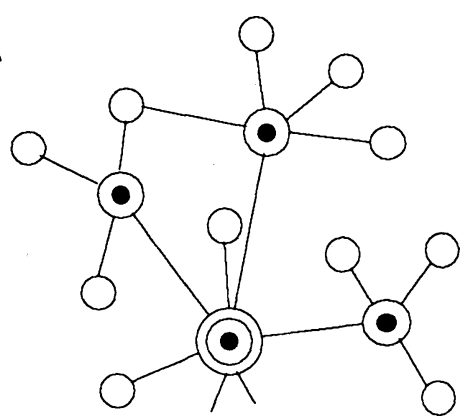

B

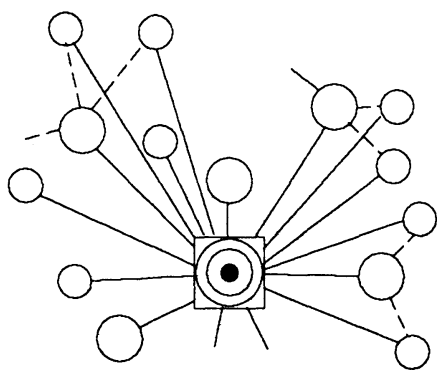

C

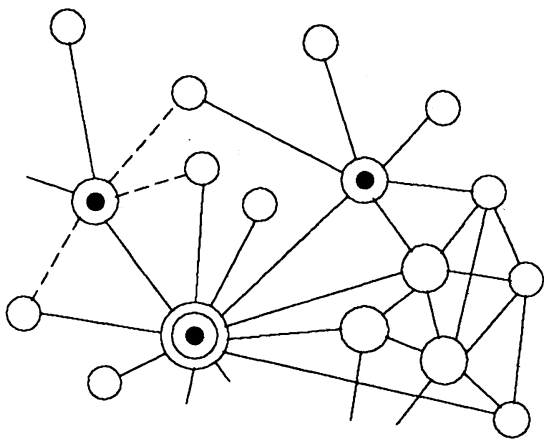

D

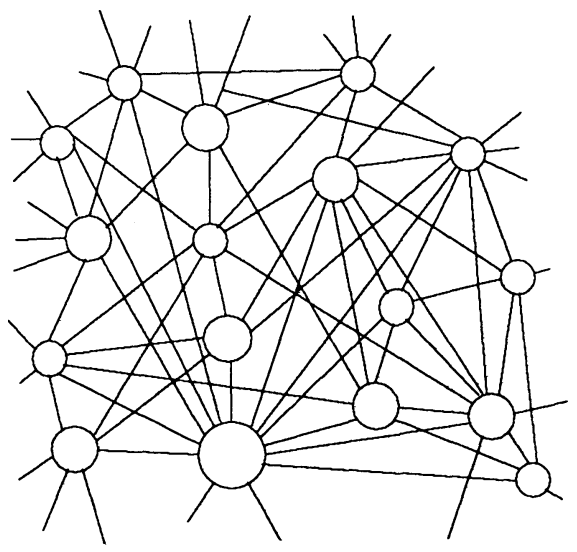

Source : Dematteis, Gambino et Coppola, (1986)

agissent dans ce cas indirectement, dans la mesure où elles favorisent «les préconditions» nécessaires pour le développement des services (soit les forces favorisant l'agglomération et autres facteurs externes).

$2^{\circ}$ Le second enseignement est plus spéculatif; il est plus lié au rapport entre le territoire et l'entreprise. Si le territoire constitue un incubateur au moins pour certaines entreprises, il devient alors quelque chose de plus que «l'environnement» évoqué par l'économie. En effet, le réseau, expression du caractère territorial croissant de l'entreprise, vit et survit justement parce que plusieurs des relations entre les entreprises ne relèvent plus uniquement des relations de marché ou des relations strictement technologiques. Mais cela est difficile à comprendre et à définir conceptuellement. 
Le caractère plutôt flou de ce territoire de l'entreprise s'explique par la façon dont cette dernière use des ressources de l'espace. Dans certains cas, l'entreprise a la capacité de faire acquérir de la valeur et de la signification à des composantes territoriales déterminées en les modelant et les remodelant constamment; dans d'autres, l'entreprise est en mesure d'ignorer ces composantes territoriales, en réduisant le territoire à une simple infrastructure physique ou de support. Alors que les processus toujours plus évolués de la réorganisation industrielle s'affirment, on peut observer, en effet, comment tant les villes que les territoires périphériques développent des caractéristiques et des formes toujours plus fragmentées.

$3^{\circ}$ Un autre constat est le bien-fondé de la notion de réseau. Le réseau explique bien les problématiques du polyformisme des entreprises et du territoire. Ces réseaux d'entreprises et de territoires ne sont pourtant pas des entités identiques. Les premiers modèlent les seconds, pas tellement en fonction de leurs nombreuses relations, mais par la stabilité et la synergie qu'ils génèrent dans ces relations. Ce «façonnage» des réseaux d'entreprises sur les réseaux territoriaux se réalise lorsque les premiers contribuent à la spécialisation, à la caractérisation et à la différentiation stable et structurelle des noeuds territoriaux.

$4^{\circ}$ La notion de réseau peut changer non seulement selon des phénomènes étudiés, mais aussi à l'intérieur d'un même phénomène selon les variables et les représentations employées. Alors que pour les spécialistes du territoire, les interconnexions s'expliquent par les infrastructures et donc, avant tout, par les relations matérielles entre les noeuds, pour les spécialistes de l'organisation industrielle il est dû, avant tout, aux échanges non matériels, où le réseau relationnel est le plus souvent virtuel, de type télématique et informatique. Par conséquent, on trouve souvent des évaluations différentes et antithétiques à propos des possibilités réelles des noeuds à appartenir à un réseau donné.

$5^{\circ}$ Enfin, le réseau peut être reproduit à travers des variables de complexité et de globalisation. L'espace structuré par un réseau implique en effet l'existence de vides; là où deux segments interreliés se croisent un nouveau noeud peut naitre. Cette possibilité théorique semble se concrétiser dans la mesure où les processus de diffusion ont créé sur le territoire des sédimentations croissantes et des «connotations historico-culturelles» capables de déterminer avec une fréquence toujours plus grande les conditions de passage d'un noeud fugitif à un noeud stable, c'est-à-dire les conditions pour générer d'autres «Troisième Italie». 


\section{BIBLIOGRAPHIE}

Aiken, M., Martinotti, G. et Strickland, D., (1987), «Sviluppo industriale e gerarchia urbana in Italia», dans Polis, no 1, p. 347-372.

Antonelli, C., (1986), L'attività innovativa in un distretto tecnologico, Fondazione Agnelli, Turin.

Arrighetti, A., (1988), «L'evoluzione dell'impresa manifatturiera alla luce della categoria della flessibilitàs, dans M. Righini (éd), La sfida della flessibilità, F. Angeli, Milan, p. 7087.

Baldisseri, R., (1984), «Decentramento produttivo e ristrutturazione industriale», Economia e Politica Industriale, p. 165-180.

Bernardi, R., (1988), «La struttura urbana del Veneto», (éd.) C.N.R. Groupe coordonné de géographie humaine, Rome.

Borlenghi, E. et Dematteis, G., (1982), «Localizzazione industriale e politica territoriale nell' area torinese», dans Torino-Detroit : due città a confronto, Séminaire d'étude sur «la crise économique et la réponse politique de la ville de l'automobile», Université Harward, Centre pour les études européenres, ouvrage édité par la ville de Turin, p. 109-120.

Brunet, R., (1980), «La composition des modèles dans l'analyse spatiale», L'espace géographique, no 9, p. 253-265.

Butera F. (1986), «Nuove tecnologia ed economia della flessibilità», L'Impresa, no 2, p. 43 50.

Butera F., (1988), «La flessibilità : da fonte di deregolamentazione a componente di nuovi modelli di regolamento nelle organizzazioni» dans M. Regini (éd). La sfida della flessibilità, F. Angeli, Milan, p. 55-69.

Camagni, R. et Rabelloti, R., (1988), «L'innovazione macro-organizzativa nel settore tessileabbigliamento", Sviluppo e Organizzazione, no 108, p. 2-8.

Camagni, R. et Gambarotto, F., (1988), «Gli accordi di cooperazione come nuove forme di sviluppo estemo delle imprese», Economia e politica industriale, no 58, p. 94-138.

Camagni, R., (1989), Economia urbana, La nuova Italia Scientifica, Rome.

Cappelin, R., (1989), «Networks nelle città e network tra città» dans F. Curtis et L. Diappi (éd.) Gerarchie e reti di città : tendenze e politiche, F. Angeli, Milan, p. 72-97.

Celant, A., (1988), "Nuova città e nuova campagna» dans A. Celant (éd.), Città e nuova campagna. L'Italia nella transizione, Patron, Bologne.

Conti S. et Spriano, G., (1989), Urban structures, technological innovation and international metropolitan networks, Fondazione Agnelli, Turin.

Cori, B., (1985), «Diversificazioni territoriali e settoriali nello sviluppo del sistema imprenditoriale italiano» dans ASSEFOR Nuove imprese. Una risorsa strategica dell' economia italiana, Maggioli, Bologne, p. 15-67.

Costa, P., (1986), «Lo sviluppo economico italiano tra l'uno e il molteplice», Studi e notizie ILRES, no 17, p. 9-12.

Da Pozzo, C., (1979), «Città e industria, società e territorio» dans AGEI (éd.), Studi su città, sistemi metropolitani, sviluppo regionale, Giardini Editori, Pise.

Dematteis, G., Gambino, R. et Coppola, P., (1986), Città e territorio in Italia negli anni ' 80 Prima analisi delle tendenze, dei problemi e delle politiche, C.N.R. Projet sur «La structure à l'évolution de l'économie italienne», sous-projet no 4 , thème 8 , cahier no 2 , Turin.

Dematteis, G., (1989a), «Modellin urbani a rete. Considerazioni preliminari» dans F. Curti et L. Diappi (éd.), Gerarchie e reti di citttà : tendenze e politiche, F. Angeli, Milan, p. 27 48. 
Dematteis, G., (1989b), «Contingenza ambientale e ordine economico, lo sviluppo locale in una prospettiva geografica», dans C. Beccattini (éd.), Modelli locali di sviluppo, Il Mulino, Bologne, p. 131-147.

Emanuel, C., (1989), Le trasformazioni recenti delle reti urbane nella Padania Centrooccidentale, CNR, op. cit., sous-projet 4 , Thème 8 , Cahier 8 , Turin.

Esposito, E., Raffa, M. et Zolio, G., (1989), «Reti di impresa e impresa a rete», Sviluppo e Organizzazione, no 114, p. 87-103.

Folloni, G., (1984), «La struttura settoriale dell'economia italiana : un'analisi 1965-1981, Economia e politica industriale, no 41, p. 183-206.

Garofoli, G., (1986), «I sistemi produttivi locali in Lombardia : meccanismi di funzionamento e politiche di intervento» dans U. Leone (éd.), La rivalorizzazione territoriale in Italia, F. Angeli, Milan, p. 83-110.

Garofoli, G., (1989), «Formazione di nuove imprese e sviluppo locale» dans C. Antonelli, R. Cappelin, R. Jannaccone et R. Pazzi, (éd.), Le politiche di sviluppo locale, nuove imprese, innovazione e servizi alla produzione, F. Angeli, Milan, p. 37-91.

Giacometti, M., (1986), «Tipologie e funzioni di alcune politiche territoriali per l'innov azione», Economia Pubblica, no 5, p. 577-584.

Landini, P. C., (1987), «Armatura urbana, strutture territoriali, processi di regionalizzazione» dans Atlante economico e commerciale delle regioni italiane, Somea, Rome.

Lito Newsletter no 1, (1989), Impresa-rete : modello per gli anni 90?, (édité par B. CROCE et C. Terraccion), p. 1-8.

Lorenzoni, G., (1985), «Dalla singola impresa agli aggregati di imprese : la costellazione», dans V. BALLONI (éd.) Esperienze di ristrutturazione industriale, Il Mulino, Bologne, p. 41-50.

Maillat, D., (1989), «Piccole e medie imprese, innovazione e sviluppo territoriale», Studi e Informazioni, no 3, p. 85-99.

Petsimeris, P., (éd.) (1989), Le reti urbane tra decentramento e centralità. Nuovi aspetti della geografia della città, Angeli, Milan.

Regini, M. et Sabel, C. F., (1989), «Le strategie di riaggiustamento in Italia : uno sguardo d'insieme in chiave comparata» dans M. Regini et C.F. Sabel (éd.) Strategie di riaggiustamento industriale, Il Mulino, Bologne, p. 11-60.

Senn, L., (1988), «Nuove prospettive di politica economica alla luce delle trasformazioni terziarie» dans Il sistema terziario in Italia, cahier de recherche de politique commerciale et touristique, no 30, ISCOM, Rome, p. 197-200.

Senn, L., (1989), L'impatto territoriale dei processi di aggiustamento, Institut universitaire de Bergamo, Cahier de recherche, juillet.

Tassinari, G., (1984), Le trasformazioni dell' industria italiana negli anni 70. Analisi dei risultati censurai, CLUEB, Bologne.

Vaccà, S., (1985), «Piccola e grande impresa in une fase di rivoluzione scientifico tecnologica», Economia e politica industriale, no 47, p. 27-49.

Vallega, A., (1978), Regione e territorio, Mursia, Milan. 